\title{
Wandering consolidation
}

\author{
Michael AR Keane, David M Hansell, Charles RK Hind
}

A 63-year-old man who had previously been fit and well, developed an acute illness with headaches and fever. His chest X-ray is shown in figure 1 . Other investigations revealed an elevated lactate dehydrogenase and gamma glutamyl transferase and transient microscopic haematuria for which no cause was found. Following antibiotic treatment, his symptoms settled. Over the next six weeks he complained of increasing breathlessness but had no other symptoms. His family doctor found signs of left lower lobe consolidation and treated him with antibiotics, but there was no symptomatic improvement and he was referred to hospital. It was noted that he had travelled to Canada, Fiji, Australia, and Singapore a year previously.

On examination he appeared unwell and he had signs of left-sided consolidation. He was in atrial fibrillation and was normotensive. Routine blood tests were normal other than an erythrocyte sedimentation rate of $75 \mathrm{~mm} / \mathrm{h}$. His repeat chest X-ray is shown in figure 2 .

Royal Brompton Hospital, London SW3 6NP, UK

MAR Keane

DM Hansell

Royal Liverpool

University Hospital, Liverpool L7 8XP, UK CRK Hind

Correspondence to Dr DM Hansell

Accepted 3 May 1995

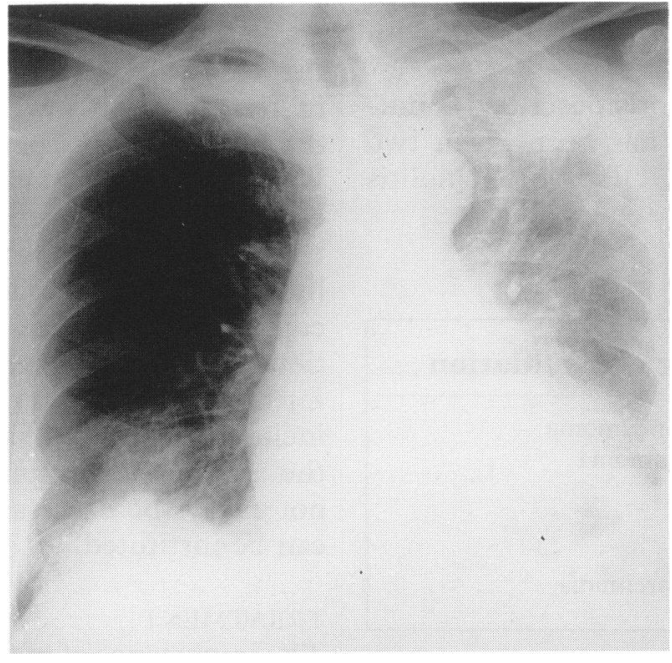

Figure 1 Initial chest X-ray

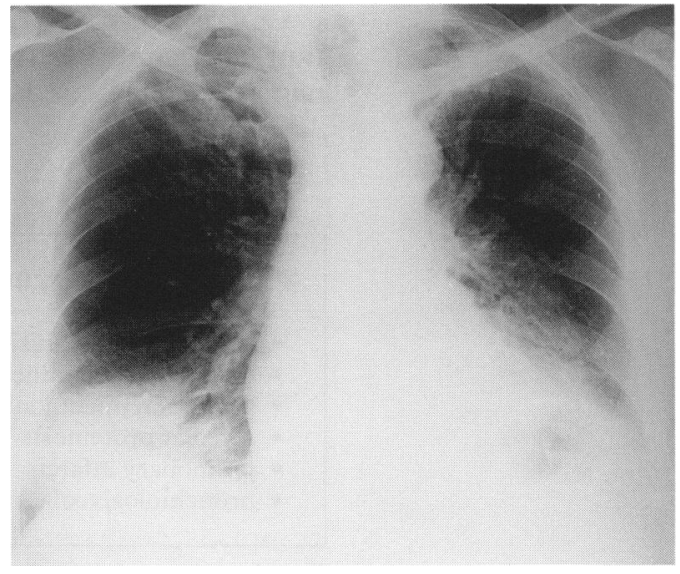

Figure 2 Chest X-ray six weeks later

\section{Questions}

1 What is the most likely diagnosis?

2 Suggest three alternative diagnoses. 


\section{Answers}

QUESTION 1

Cryptogenic organising pneumonia (COP). The initial chest X-ray (figure 1) shows left apical and basal consolidation and a small area of consolidation at the right base. The leftsided consolidation improved over the succeeding six weeks, but at the same time the right basal shadowing worsened (figure 2).

QUESTION 2

Although there is a long list of causes of multifocal consolidation on a chest X-ray, causes of migratory consolidations, ie, new areas of abnormality appearing whilst other areas resolve, are relatively few (box 1). ${ }^{1}$

\section{Discussion}

In this case the absence of peripheral eosinophilia, aspiration, or other relevant history make cryptogenic organising pneumonia the most likely diagnosis. A trucut biopsy was subsequently obtained using computed tomographic (CT) guidance and histology confirmed this. He responded well to treatment with prednisolone.

COP is a relatively recently described condition but, confusingly, it has been given two names: $\mathrm{COP}^{2}$ and $\mathrm{BOOP}^{3}$ (bronchiolitis

\begin{tabular}{|l|}
\hline Causes of migrating consolidation \\
\hline - cryptogenic organising pneumonia \\
- chronic eosinophilic pneumonia \\
- aspiration pneumonia \\
- pulmor proteinosis \\
- bronchioloalveolar cell carcinoma \\
\hline
\end{tabular}

Box 1

\begin{tabular}{|l|}
\hline COP: associations \\
\hline - rheumatoid arthritis \\
- ulcerative colitis \\
- immunosuppression (eg, heart-lung \\
transplantation) \\
- drugs: amiodarone, acebutolol \\
- radiation pneumonitis \\
\hline
\end{tabular}

Box 2

1 Hansell DM. What are BOOP and COP? Clin Radiol 1992; 45: 369-70.

2 Davison AG, Heard BE, McAllister WAC, TurnerWarwick MEH. Cryptogenic organizing pneumonitis. $Q \mathcal{f}$ Med 1983; 207: 382-94.

3 Epler GR, Colby TV, McLoud TC, Carrington CB, Gaensler EA. Bronchiolitis obliterans organizing pneumonia. $N$ Engl f Med 1985; 312: 152-8. obliterans organising pneumonia) which have come to be used interchangeably. An organising pneumonia occurs when inflammation in the distal lung structures incompletely resolves and in the past was most commonly seen as a sequel to bacterial pneumonia. However, although a number of causes are now recognised (box 2), in practice, the majority of cases are truly cryptogenic. Organising pneumonia is perhaps best considered a response of the lung to injury by a variety of different agents. By definition, no identifiable agent, infective or otherwise, is present in cases of COP.

The term BOOP was coined by the original American authors because the respiratory bronchioles may become blocked by buds of immature granulation tissue which originate in the alveoli. This terminology is unfortunate as it can cause confusion with obliterative bronchiolitis, an entirely different disease affecting the small airways.

CLINICAL AND RADIOLOGICAL FEATURES OF COP

The usual clinical presentation of COP is rather non-specific with cough, fever, malaise, and dyspnoea. The radiology is exemplified by this case with peripheral consolidations, often in the mid and lower zones, appearing and disappearing over a period of weeks or months. ${ }^{4}$ Uncommonly, cavitation and pleural effusions occur. There has been a report of COP presenting seasonally, associated with disturbance of liver function. ${ }^{5}$ CT adds little, other than to confirm the presence of multifocal consolidations but can be useful to identify a suitable site for biopsy. However, given a suitable history, including a lack of response to antibiotics, and the typical radiographic findings, biopsy may not always be necessary and a trial of steroids can be instituted. ${ }^{6}$

\section{TREATMENT}

The importance of making the diagnosis is that the condition is usually very sensitive to steroids with complete clinical and radiological remission being the rule, although relatively high doses of steroids may have to be continued for several months.

\section{Final diagnosis}

\section{Cryptogenic organising pneumonia}

Keywords: crytogenic organising pneumonia, chest $\mathrm{X}$-ray

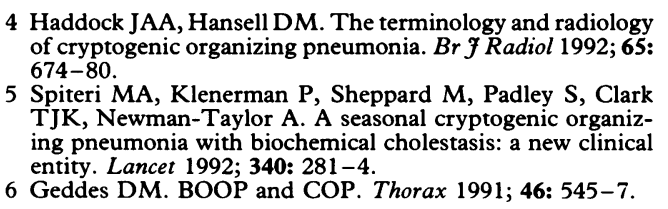

4 Haddock JAA, Hansell DM. The terminology and radiology of crypt
$674-80$

5 Spiteri MA, Klenerman P, Sheppard M, Padley S, Clark in , Newman-Taylor A. A seasonal cryptogenic organizentity. Lancet 1992; 340: 281-4.

6 Geddes DM. BOOP and COP. Thorax 1991; 46: 545-7. 